\title{
Nuevo sistema de predicción de dificultad en la ventilación a través de dispositivos supraglóticos
}

Artículo original: T. Saito, S. T. H. Chew, W. L. Liu. A proposal for a new scoring system to predict difficult ventilation through a supraglottic airway device. British Journal of Anaesthesia, 2016; 117 (S1): 83-86 ( $\underline{\text { PubMed) }}$ (HTML)

Vicente Orgaz M, González Velasco R, García Rueda A, Rodríguez Bertos C.

\section{Hospital Universitario Severo Ochoa. Leganés, Madrid.}

\section{Resumen}

El objetivo de este estudio fue proponer y validar un nuevo sistema de puntuación clínica para predecir la dificultad de ventilación a través de un dispositivo supraglótico (DSG).

Los autores desarrollaron un score que se validó prospectivamente en 5532 pacientes. Se asignaron valores numéricos a cada uno de los 4 factores de riesgo que se identificaron: varón, mayor de 45 años, distancia tiromentoniana (DTM) corta y movilidad cervical limitada, y su suma compuso el score.

El rango de dicho score va de 0 a 7 puntos. Un score de 4 o mayor se asocia a un riesgo incrementado de dificultad de ventilación a través de DSG.

Nuestra conclusión es que el nuevo score para predicción de dificultad de ventilación a través de dispositivos supraglóticos es fácil de usar y podría ayudar en el plan de manejo de la VAD por los anestesiólogos.

\section{Introducción}

El objetivo de este estudio fue proponer y validar un nuevo sistema de puntuación clínica para predecir la dificultad de ventilación a través de un dispositivo supraglótico (DSG).

Los autores desarrollaron un score que se validó prospectivamente en 5532 pacientes. Se asignaron valores numéricos a cada uno de los 4 factores de riesgo que se identificaron: varón, mayor de 45 años, distancia tiromentoniana (DTM) corta y movilidad cervical limitada, y su suma compuso el score.

El rango de dicho score va de 0 a 7 puntos. Un score de 4 o mayor se asocia a un riesgo incrementado de dificultad de ventilación a través de DSG.
Nuestra conclusión es que el nuevo score para predicción de dificultad de ventilación a través de dispositivos supraglóticos es fácil de usar y podría ayudar en el plan de manejo de la VAD por los anestesiólogos.

Las últimas revisiones de las guías de vía aérea difícil (VAD) disponibles (1, 2), posicionan a los dispositivos supraglóticos (DSG), como dispositivos de rescate cuando surgen problemas durante la ventilación con mascarilla facial o en la intubación endotraqueal.

Pero aun así, ningún plan de vía aérea está libre de fracasar. De ahí la importancia de anticiparnos a las dificultades que pudieran surgir en determinados pacientes con estos dispositivos.

El fallo en la inserción de un DSG está en torno a $0,5-4,7 \%$ (3). Son causas 
conocidas las anomalías anatómicas, la inflamación de la vía aérea superior o el sangrado tras los repetidos intentos de intubación.

El objetivo del estudio fue proponer y validar un nuevo test clínico de predicción de dificultad en la ventilación a través de DSG.

\section{Resumen}

Para el análisis, los autores hicieron un primer estudio con una población de 14.480 pacientes mayores de 18 años que fueron intervenidos bajo anestesia general utilizando algún dispositivo supraglótico. Se usaron diferentes tipos de mascarillas laríngeas (ML), entre las cuales podemos destacar: ML clásica, ML Proseal, ML Supreme y la ML Fastrach (4).

Para su estudio, definieron la dificultad de ventilación a través de DSG como la incapacidad de aportar una adecuada oxigenación durante la inducción de la anestesia general debido a una o más de las siguientes causas:

- Sellado inadecuado de la mascarilla.

- Exceso de fuga.

- Excesiva resistencia a la entrada y salida del gas.

Consideraron además como signos de ventilación inadecuada:

- Ausencia de elevación torácica.

- Ausencia de ruidos respiratorios.

- Auscultación pulmonar con signos de obstrucción severa.

- Cianosis.

- Distensión gástrica.

- Desaturación.
- Capnografía ausente o inadecuada.

- Valores espirométricos inadecuados.

- Cambios hemodinámicos asociados a la hipoxemia o hipercapnia.

En esa población se identificaron 74 pacientes cuya ventilación a través de estos dispositivos resultó difícil.

Posteriormente, tras realizar un análisis multivariante concluyeron que existen cuatro factores de riesgo para la dificultad de ventilación con DSG y asignaron a cada uno de ellos, una puntuación (Tabla 1):

\begin{tabular}{|l|l|}
\hline SEXO MASCULINO & 1 PUNTO \\
\hline $\begin{array}{l}\text { EDAD MAYOR DE 45 ANOS } \\
\text { MOVILIDAD CERVICAL } \\
\text { LIMTIADA }\end{array}$ & 1 PUNTO \\
\hline $\begin{array}{l}\text { DISTANCIA TIROMENTONIANA } \\
\text { CORTA (K5.5) }\end{array}$ & 3PUNTOS \\
\hline $\begin{array}{l}\text { Tabla 1. EScala de predicción de dificultad de } \\
\text { ventilación a través de DSG (4) }\end{array}$
\end{tabular}

Se obtuvo, por tanto, una escala en el que la suma de todos los puntos posibles daba un rango de 0 a 7 . Todos ellos con una $\mathrm{P}<0,05$ y por lo tanto estadísticamente significativos.

A continuación, esta escala se validó en una nueva cohorte de 5532 pacientes que cumplían los criterios de inclusión entre las fechas de noviembre de 2013 a abril de 2014.

Los resultados que se observaron fueron que, dentro de la cohorte de 5532 pacientes, 22 tuvieron dificultad para la ventilación.

Cuando estratificaron a los pacientes, encontraron que en el grupo de "alto riesgo" tuvieron 6 veces más riesgo de dificultad de ventilación por DSG comparados con aquellos del grupo de "bajo riesgo". (Tabla 2).

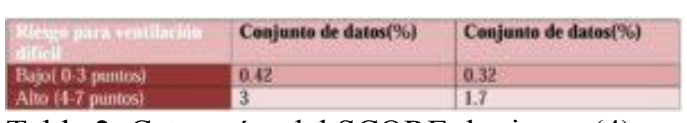

Tabla 2. Categorías del SCORE de riesgo (4). 
La sensibilidad de la escala de riesgo fue del $23 \%$ mientras que la especificidad fue del $95 \%$, resultando un valor predictivo negativo del $99.6 \%$. Este hecho, nos indica que los pacientes que no cumplen los factores de riesgo o tienen un 'riesgo bajo' tienen muy poca probabilidad de presentar complicaciones en la ventilación con algún dispositivo supraglótico.

\section{Comentario}

Los DSG ocupan un papel principal en las nuevas guías para resolver un fallo de intubación. Incluso en poblaciones seleccionadas como en la paciente obstétrica, el DSG se ha convertido en una pieza fundamental en los planes de aislamiento de la vía aérea cuando surgen dificultades.

Las guías DAS del 2015 están de acuerdo en enfatizar que el objetivo principal debe ser la oxigenación del paciente más que la intubación.

Por ello, es muy importante detectar antes de la inducción a los pacientes que puedan presentar dificultades para ventilarse a través de DSG y así poder elaborar nuestra estrategia correctamente, escogiendo los planes con mayor probabilidad de éxito. Hoy en día, hay numerosas cirugías que se realizan con un DSG como primera opción, por lo que estas escalas que predicen el posible fallo de estos dispositivos nos ayudarían también a inclinar la balanza por la intubación endotraqueal en estas circunstancias.

Actualmente, existen diferentes métodos que intentan predecir la dificultad en la vía aérea tanto para la ventilación como para la intubación.

Para los DSG se contaba con métodos poco rigurosos, cuya eficacia $\mathrm{y}$ efectividad distan mucho de estar aseguradas. Uno de estos es la ayuda del acrónimo RODS (5) para la predicción de una posible dificultad en la ventilación a través de estos dispositivos.

\section{RODS:}

\section{- R: RESTRICTED MOUTH}

OPENING: Distancia interincisivos menor de 2'5 $\mathrm{cm}$ puede conllevar dificultad.

\section{- O: OBSTRUCCIÓN VÍA AÉREA}

\section{- D: DISTORSIÓN DE LA VÍA}

AÉREA: El dispositivo supraglótico no proporcionará un sellado adecuado, no se podrá intubar a su través al no confrontar la salida de la mascarilla con la abertura glótica.

- S: STIFF. RIGIDEZ: Rigidez pulmonar o cervical. La primera provocará presiones pico elevadas que puede superar la presión de sellado de la mascarilla y la segunda dificultad de colocación.

El presente artículo abre el campo de la predicción sobre el uso de los DSG, que son el dispositivo de rescate de referencia ante una vía aérea difícil no prevista, pero que también pueden darnos problemas a la hora de ventilar a algunos de nuestros pacientes.

Con la escala propuesta se pueden identificar a los pacientes con bajo o alto riesgo. Un paciente con puntuación de 4 o mayor tiene el riesgo de dificultad de ventilación a través de un DSG aumentado de 6 a 7 veces.

Podemos encontrar limitaciones en el estudio como la variabilidad de dispositivos supraglóticos que se utilizaron, lo cual depende mucho de la preferencia del anestesiólogo y de su destreza con cada uno de ellos. Además, la mayoría de los pacientes incluidos en este estudio fueron de raza asiática, que 
pueden tener diferente anatomía de la vía aérea respecto a los no asiáticos.

Por ello consideramos conveniente que se realicen más estudios similares a este en otras etnias y en cohortes mayores.

Creemos que esta escala podría llegar a tener utilidad. Nos parece una manera rápida y sencilla de predecir una posible dificultad en la ventilación de nuestros pacientes con DSG que puede ponernos en alerta para optar por otra estrategia, o por el contrario, tener la seguridad de que, teniendo un riesgo bajo, la ventilación de nuestro paciente no se verá comprometida.

\section{Conclusión}

La principal fortaleza de estudios como éste, recae en conocer que existe la posibilidad de fracaso incluso manejando de manera correcta los diferentes planes de rescate para la vía aérea difícil. Por ello, cuanta más información dispongamos a la hora de diseñar nuestra estrategia, más seguros estaremos de llegar al éxito con ella. (Figura 1).

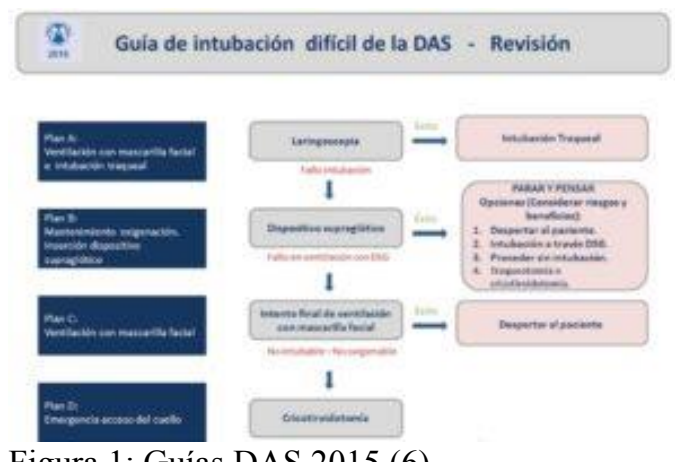

Figura 1: Guías DAS 2015 (6)

\section{Bibliografía}

- Apfelbaum JL, Hagberg CA, Caplan RA, Blitt CD, Connis RT, Nickinovich DG, et al. Practice guide- lines for management of the difficult airway: an updated re- port by the American Society of Anesthesiologists Task Force on Management of the Difficult Airway. Anesthesiology 2013; 118: 251-70. (ubMed) (HTML)

- Frerk C, Mitchell VS, McNarry AF, Mendonca C, Bhagrath R, Patel A, et al. Difficult Airway Society 2015 guidelines for management of unanticipated difficult intubation in adults. Br J Anaesth 2015; 115: 82748. (ubMed) ( $\underline{\text { HTML) }}$ (PDF)

- Cook TM, Trumpelmann P, Beringer R, Stedeford J. A randomised comparison of the Portex Softseal laryngeal mask airway with the LMA-Unique during anaesthesia. Anaesthesia 2005; 60: 1218-25. ( PubMed)

- Saito T, Liu W, Chew ST, Ti LK. Incidence of and risk factors for difficult ventilation via a supraglottic airway device in a population of 14,480 patients from South-East Asia. Anaesthesia 2015; 70: 1079-83. (PubMed)

- Hung O, Murphy M. "Changing practice in airway management: are we there yet?." Can J Anaesth 2004 Dec; 51 (10): 963-8. (ubMed)

- Anon, (n.d.). Resumen de las Guias DAS 2015 - anestesiar.org. [online] Available at: https://anestesiar.org/2015/resumen-de-lasguias-das-2015/ [Accessed 14 Mar. 2018]. (HTML)

Correspondencia al autor

Marta Vicente Orgaz

martavicenteorgaz@hotmail.com

Médico Interno Residente. Servicio de Anestesiología y Reanimación.

Hospital Universitario Severo Ochoa. Leganés, Madrid.

Aceptado para blog en agosto de 2018. 